\title{
Differentiation between Oligodendroglioma Genotypes Using Dynamic Susceptibility Contrast Perfusion-Weighted Imaging and Proton MR Spectroscopy
}

\author{
S. Chawla, J. Krejza, A. Vossough, Y. Zhang, G.S. Kapoor, S. Wang, D.M. O'Rourke, E.R. Melhem, and H. Poptani
}

\begin{abstract}
BACKGROUND AND PURPOSE: Oligodendrogliomas with 1p/19q chromosome LOH are more sensitive to chemoradiation therapy than those with intact alleles. The usefulness of dynamic susceptibility contrast-PWI-guided ' $\mathrm{H}$-MRS in differentiating these 2 genotypes was tested in this study.
\end{abstract}

\begin{abstract}
MATERIALS AND METHODS: Forty patients with oligodendrogliomas, 1p/19q LOH $(n=23)$ and intact alleles $(n=17)$, underwent MR imaging and 2D-'H-MRS. ${ }^{1} \mathrm{H}-\mathrm{MRS}$ VOI was overlaid on FLAIR images to encompass the hyperintense abnormality on the largest crosssection of the neoplasm and then overlaid on CBV maps to coregister CBV maps with ${ }^{~} \mathrm{H}-\mathrm{MRS}$ VOI. $\mathrm{rCBV}_{\text {max }}$ values were obtained by measuring the CBV from each of the selected ${ }^{~} \mathrm{H}-\mathrm{MRS}$ voxels in the neoplasm and were normalized with respect to contralateral white

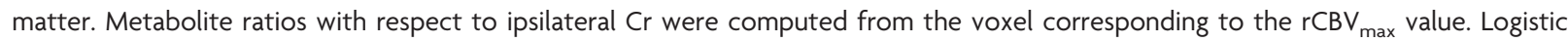
regression and receiver operating characteristic analyses were performed to ascertain the best model to discriminate the 2 genotypes of oligodendrogliomas. Qualitative evaluation of conventional MR imaging characteristics (patterns of tumor border, signal intensity, contrast enhancement, and paramagnetic susceptibility effect) was also performed to distinguish the 2 groups of oligodendrogliomas.
\end{abstract}

RESULTS: The incorporation of $\mathrm{rCBV}_{\max }$ value and metabolite ratios (NAA/Cr, Cho/ Cr, Glx/Cr, myo-inositol/Cr, and lipid + lactate/Cr) into the multivariate logistic regression model provided the best discriminatory classification with sensitivity (82.6\%), specificity (64.7\%), and accuracy $(72 \%)$ in distinguishing 2 oligodendroglioma genotypes. Oligodendrogliomas with $1 \mathrm{p} / 19 \mathrm{q}$ LOH were also more associated with paramagnetic susceptibility effect $(P<.05)$.

CONCLUSIONS: Our preliminary results indicate the potential of combing PWI and ${ }^{1} \mathrm{H}-\mathrm{MRS}$ to distinguish oligodendroglial genotypes.

ABBREVIATIONS: $\mathrm{Glx}=$ glutamate + glutamine; $\mathrm{LOH}=$ loss of heterozygosity; $\mathrm{rCBV}=$ relative cerebral blood volume; $r \mathrm{CBV}$ max $=$ maximum $\mathrm{rCBV} ; \mathrm{WHO}=\mathrm{World}$ Health Organization

O ligodendroglial neoplasms account for $5 \%-18 \%$ of all gliomas. ${ }^{1}$ These neoplasms are typically classified into grade II and III oligodendrogliomas according to the WHO classification system. However, this classification is not sensitive and specific for predicting response to therapy and overall survival duration. ${ }^{2}$ Emerging molecular abnormalities in these neoplasms have shown promise in an accurate diagnosis, which has led to improved strategies in management of specific subtypes. ${ }^{3}$ The characteristic feature of oligodendrogliomas at the molecular level is loss of the shorter arm of chromosome 1 (1p) or codeletion of $1 \mathrm{p}$ and the longer arm of chromosome 19 mutation that harbors

Received May 15, 2012; accepted after revision August 31.

From the Departments of Radiology (S.C., J.K., A.V., Y.Z., S.W., E.R.M., H.P.) and Neurosurgery (G.S.K., D.M.O.), University of Pennsylvania, Philadelphia, Pennsylvania.

Please address correspondence to Harish Poptani, PhD, University of Pennsylvania, B6 Blockley Hall, 423 Guardian Dr, Philadelphia, PA 19104; e-mail: Harish.Poptani@ uphs.upenn.edu

http://dx.doi.org/10.3174/ajnr.A3384 tumor suppressor genes. Whereas isolated $19 \mathrm{q} \mathrm{LOH}$ occurs in astrocytomas, $1 \mathrm{p} \mathrm{LOH}$ or combined $1 \mathrm{p} / 19 \mathrm{q} \mathrm{LOH}$ is uncommon in gliomas other than oligodendrogliomas and oligoastrocytomas. ${ }^{4,5}$ Molecular classification in oligodendrogliomas correlates better with clinical outcome compared with the WHO classification system. ${ }^{6}$ Several clinical studies, including phase III trials, have reported that patients with oligodendroglioma harboring $1 \mathrm{p} / 19 \mathrm{q} \mathrm{LOH}$, regardless of the higher tumor grade, demonstrate better prognosis and response to chemoradiation therapy than patients with intact $1 \mathrm{p} / 19 \mathrm{q}$ alleles. ${ }^{7-9}$ Furthermore, patients with $1 \mathrm{p} / 19 \mathrm{q}$ LOH exhibit prolonged progression-free survival and overall survival duration subsequent to chemoradiation therapy. ${ }^{6}$ Thus, differentiation between the 2 genotypes, before initiation of treatment, is of paramount importance because treatment strategy is different.

PWI with dynamic susceptibility-weighted contrast-enhanced imaging and ${ }^{1} \mathrm{H}-\mathrm{MR}$ spectroscopy have been used to distinguish genotypic profiles of oligodendrogliomas. ${ }^{10-12}$ Although promis- 
ing results were reported in the differentiation of $\mathrm{LOH}$ in grade II oligodendrogliomas, there was a substantial overlap in patients with grade III oligodendrogliomas. ${ }^{1,12}$ Using ${ }^{1} \mathrm{H}-\mathrm{MR}$ spectroscopy, Jenkinson et $\mathrm{al}^{10}$ reported a higher $\mathrm{Cho} / \mathrm{Cr}$ ratio in oligodendrogliomas with $1 \mathrm{p} / 19 \mathrm{q}$ deletion than in those with intact alleles; however, the difference was not significant.

Combined use of PWI and ${ }^{1} \mathrm{H}$-MR spectroscopy may improve the overall reliability of these advanced MR techniques in preoperative genetic profiling of oligodendrogliomas. Some investigators have used both PWI and ${ }^{1} \mathrm{H}-\mathrm{MR}$ spectroscopy to grade oligodendrogliomas. ${ }^{13}$ However, the accuracy of differentiation was poor because the selection of the ${ }^{1} \mathrm{H}-\mathrm{MR}$ spectroscopy voxel was random, regardless of underlining rCBV values. High rCBV is a surrogate marker of neoangiogenesis and is associated with increased mitotic activity. ${ }^{14,15}$ Thus, we hypothesize that ${ }^{1} \mathrm{H}-\mathrm{MR}$ spectroscopy sampling of the "islands" of high rCBV, within the neoplasm, might aid in the selection of the optimal ${ }^{1} \mathrm{H}$ - MR spectroscopy voxel, which in turn might improve the discriminative performance of ${ }^{1} \mathrm{H}$-MR spectroscopy. In accordance with this hypothesis, Chawla et $\mathrm{al}^{16}$ reported significantly higher Cho/Cr and lipid + lactate/Cr from high-grade oligodendrogliomas than in low-grade neoplasms when ${ }^{1} \mathrm{H}$-MR spectroscopy voxels were sampled from regions of high cerebral blood flow. Thus in our current study, we investigated whether ${ }^{1} \mathrm{H}-\mathrm{MR}$ spectroscopy voxels guided by dynamic susceptibility contrast-PWI will also be useful in discriminating oligodendrogliomas with $1 \mathrm{p} / 19 \mathrm{q} \mathrm{LOH}$ from oligodendrogliomas with intact alleles.

\section{MATERIALS AND METHODS Patients}

The study was approved by the Institutional Review Board and was compliant with the Health Insurance Portability and Accountability Act. Histopathologically confirmed oligodendrogliomas or mixed oligoastrocytomas, according to the WHO classification system, ${ }^{2}$ were included in the study. Because mixed oligoastrocytomas have a prominent composition of oligodendroglial cells, they were included in the oligodendroglioma group. Only treatment-näive patients were included, and patients with prior biopsy or surgery were excluded. Histopathologic diagnosis was obtained in all patients by surgical resection or biopsy after the imaging study. Cytogenic profile was obtained either by fluorescent in situ hybridization on fresh tumor specimens or polymerase chain reaction on paraffin-embedded specimens. Fifty patients with oligodendroglioma were recruited from July 2006 to September 2010. Six patients who did not have a cytogenic profile were excluded from the study. In addition, 4 patients were excluded from the analysis because of suboptimal PWI or ${ }^{1} \mathrm{H}-\mathrm{MR}$ spectroscopy data quality. Data analysis was performed on the remaining 40 patients [average age, $45.24 \pm 14.04$ years; 22 men and 18 women]. These patients were classified into 2 groups: patients with $1 \mathrm{p}$ or $1 \mathrm{p}$ and $19 \mathrm{q} \mathrm{LOH}(n=23)$, and patients with intact alleles $(n=17)$. Thirteen of 23 patients with $1 \mathrm{p} / 19 \mathrm{q} \mathrm{LOH}$ oligodendrogliomas had low-grade tumors (WHO grade II), and 10 had high-grade tumors (WHO grade III). Among the 17 oligodendrogliomas with intact alleles, 9 were of low grade and 8 were of high grade.

\section{Fluorescence In Situ Hybridization}

Surgical specimens were dissociated in tissue culture medium and directly processed for interphase fluorescent in situ hybridization analysis. The probes for 1p36 (PAX7) and 19q13.4 (CTC284K17) were labeled by nick translation with ChromoTide AlexaFluor 594-dUTP (Molecular Probes, Carlsbad, California). The BAC clone for 1q31 (CTC224I21) was labeled by nick translation with ChromoTide fluorescein-12-dUTP (Molecular Probes). The probe for 19ptel (D19S238E) was labeled with SpectrumGreendUTP (Vysis, Des Plaines, Illinois). The probes were applied to tumor cell suspension slides and codenatured at $75^{\circ} \mathrm{C}$ on an Isotemp 125D heat block (Fisher Scientific, Pittsburgh, Pennsylvania). Slides were incubated overnight at $37^{\circ} \mathrm{C}$ in a moist slide moat (Boekel Scientific, Feasterville, Pennsylvania) and were washed in $0.4 \times$ standard saline citrate solution at $73^{\circ} \mathrm{C}$ for 2 minutes followed by a 1 -minute wash in $2 \times$ standard saline citrate $/ 0.1 \%$ NP-40, and counterstained with 4,6-diamidino-2-phenylindole (Sigma Chemical, St. Louis, Missouri). Fluorescent signals from 100 cells were evaluated at $100 \times$ with a Axioplan fluorescent microscope (Carl Zeiss Microscopy, Jena, Germany) equipped with the proper filter sets. An Applied Imaging System device (Applied Imaging, San Jose, California) was used to record images of stained cells.

\section{Polymerase Chain Reaction}

The allelic status of $1 \mathrm{p}$ or $19 \mathrm{q}$ was assessed by LOH assays in constitutional/tumor DNA pairs by use of microsatellite markers on 1p36 (D1S1608, D1S1161, and D1S1597) and 19q13 (D19S431, D19S559, and D19S601), as described previously. ${ }^{17}$ Tumor DNA was extracted from microdissected, formalin-fixed, paraffin-embedded sections and constitutional DNA from blood leukocytes or paraffin sections of the adjacent uninvolved brain.

\section{MR Imaging}

MR imaging and multivoxel 2D ${ }^{1} \mathrm{H}$-MRS studies were performed on a 3T Tim Trio MR scanner (Siemens, Erlangen, Germany) by use of a 12-channel phased array head coil. The imaging protocol included a 3-plane scout localizer, axial T1-weighted magnetization-prepared rapid acquisition of gradient echo (TR, $1620 \mathrm{~ms}$; TE, $3.9 \mathrm{~ms}$; TI, $950 \mathrm{~ms}$; matrix size, $192 \times 256$; section thickness, $1 \mathrm{~mm}$; sections per slab, 192; flip angle, $15^{\circ}$; NEX, 1; bandwidth, $150 \mathrm{~Hz} /$ pixel), an axial FLAIR image (TR, $9420 \mathrm{~ms}$; TE, $141 \mathrm{~ms}$; TI, $2500 \mathrm{~ms}$; section thickness, $3 \mathrm{~mm}$; number of sections, 60; flip angle, $170^{\circ}$; NEX, 1; bandwidth, $287 \mathrm{~Hz} /$ pixel), and an axial T2weighted image (TR, $2260 \mathrm{~ms}$; TE, 91ms; section thickness, $3 \mathrm{~mm}$; number of sections, 60). Contrast-enhanced T1-weighted magnetization-prepared rapid acquisition of gradient echo images were acquired after administration of a standard dose $(0.1 \mathrm{mmol} / \mathrm{kg})$ of gadodiamide contrast agent (Omniscan; GE Healthcare, Oslo, Norway).

\section{Perfusion-Weighted Imaging}

Dynamic susceptibility contrast-PWI was performed 5 minutes after a 3-mL preloading dose of intravenous gadodiamide contrast agent. The preloading injection was performed to reduce the effect of contrast leakage. For PWI, T2*-weighted gradient-echo EPI was performed during the first pass of a standard dose $(0.1$

AJNR Am J Neuroradiol 34:1542-49 Aug 2013 www.ajnr 
$\mathrm{mmol} / \mathrm{kg}, 5 \mathrm{~mL} / \mathrm{s}$ ) of intravenous contrast agent. ${ }^{18}$ FLAIR images were used to plan twenty 3 -mm-thick axial sections covering the neoplasm (field of view, $22 \times 22 \mathrm{~cm}^{2}$; matrix size, $128 \times 128$; in-plane resolution, $1.72 \times 1.72 \times 3 \mathrm{~mm}^{3}$; TR, $2000 \mathrm{~ms}$; TE, 45 ms; bandwidth, $1346 \mathrm{~Hz} /$ pixel; flip angle, 90 EPI factor, 128; echo spacing, 0.83). Forty-five sequential measurements were acquired for each section, with a temporal resolution of $2.1 \mathrm{~s}$.

\section{Proton MR Spectroscopy}

We performed single-section 2D multi-voxel ${ }^{1} \mathrm{H}-\mathrm{MRS}$ by using a point-resolved spectroscopy sequence after acquisition of PWI and postcontrast T1-weighted images. Sequence parameters included a TR of $1700 \mathrm{~ms}$; TE, $30 \mathrm{~ms}$; NEX, 3; field of view, $16 \times 16$ $\mathrm{cm}^{2}-20 \times 20 \mathrm{~cm}^{2}$; section thickness, $15-20 \mathrm{~mm}$; bandwidth, 1200 $\mathrm{Hz}$; matrix size, $16 \times 16$; and vector size, 1024 . The size of the ${ }^{1} \mathrm{H}$-MRS voxel varied from $10 \times 10 \times 15 \mathrm{~mm}^{3}$ (volume, $1.5 \mathrm{~cm}^{3}$ ) to $10 \times 12.5 \times 20 \mathrm{~mm}^{3}$ (volume, $2.5 \mathrm{~cm}^{3}$ ), depending on the dimensions of the neoplasm. The VOI was selected based on FLAIR images to include the largest cross-section of neoplasm and contralateral normal brain parenchyma in the same plane. Areas of scalp, skull base, and sinuses were avoided in the selection of the VOI. In addition, 8 outer volume saturation slabs (30- $\mathrm{mm}$ thick) were placed outside of the VOI to suppress lipid signals from the bone and scalp. The dataset was acquired by use of elliptic $k$-space sampling with weighted-phase encoding to reduce the acquisition time. Manual shimming was performed to achieve an optimal value of full width at half maximum of the water signal. In all cases, a shimming of $<20 \mathrm{~Hz}$ was achieved on the magnitude signal of the water resonance. Both water-suppressed and unsuppressed ${ }^{1} \mathrm{H}$-MRS spectra were acquired, and the unsuppressed water signal was used to compute metabolite concentrations.

\section{Data Analysis}

We constructed CBV maps on a Leonardo workstation (Siemens, Erlangen Germany) using a PWI task card (Massachusetts General Hospital, Boston, Massachusetts), as described previously. ${ }^{19}$ We initially overlaid ${ }^{1} \mathrm{H}-\mathrm{MR}$ spectroscopy VOI on the FLAIR images to select the voxels of interest encompassing the hyperintense abnormality on a Leonardo workstation by using the syngo software (Siemens). The ${ }^{1} \mathrm{H}-\mathrm{MR}$ spectroscopy VOI was then overlaid on the CBV maps to coregister the CBV maps and ${ }^{1} \mathrm{H}-\mathrm{MR}$ spectroscopy VOI. This method allowed us to sample CBV values only from the regions that corresponded to the ${ }^{1} \mathrm{H}-\mathrm{MR}$ spectroscopy voxels. We obtained $\mathrm{CBV}$ values by drawing regions of interest from each selected voxel based on the FLAIR image hyperintensity in each neoplasm. The size of the regions of interest was similar to the ${ }^{1} \mathrm{H}-\mathrm{MR}$ spectroscopy voxel dimensions. The regions of interest were drawn by the primary author, who has more than 10 years of experience with clinical spectroscopy, and verified by an experienced neuroradiologist ( $>10$ years of experience). Care was taken to avoid the cerebral blood vessels and hemorrhagic and cystic regions. CBV values were normalized with respect to contralateral normal-appearing white matter.

All ${ }^{1} \mathrm{H}$-MR spectroscopy data were analyzed by using a userindependent spectral fit program (LCModel; Stephen Provencher, Oakville, Ontario, Canada). ${ }^{20}$ The following metabolites were evaluated: NAA, Cr, Cho, Glx, and myo-inositol. The resonance at $1.3 \mathrm{ppm}$ was assigned to a combination of lactate and lipid and metabolites with Cramer-Rao lower bounds greater than $20 \%$ SD were excluded..$^{20}$ Metabolite concentrations were normalized with respect to the ipsilateral $\mathrm{Cr}$ for each voxel.

Because a region with $\mathrm{rCBV}_{\max }$ is generally thought to reflect tumor aggressiveness, ${ }^{21} \mathrm{NAA} / \mathrm{Cr}$, Cho/Cr, Glx/Cr, myo-inositol/ $\mathrm{Cr}$, and lipid + lactate/Cr ratios were computed from the voxel that also exhibited the $\mathrm{rCBV}_{\max }$ value from the tumor region that was encompassed within the VOI of ${ }^{1} \mathrm{H}-\mathrm{MR}$ spectroscopy.

As conventional MR imaging parameters have also been used for genotyping of oligodendrogliomas, ${ }^{22,23}$ the following imaging characteristics were also evaluated qualitatively: 1) sharp vs indistinct tumor border on T2-weighted images, 2) homogeneous vs heterogeneous tumor signal intensity on T2-weighted and T1weighted images, 3 ) presence or absence of a paramagnetic susceptibility effect on $\mathrm{T} 2$-weighted and $\mathrm{T} 2{ }^{*}$-weighted gradient-echo EPI images, and 4) presence or absence of contrast enhancement.

\section{Statistical Analysis}

Kolmogorov-Smirnov tests were used to determine data distributions. As the data did not depart from Gaussian distribution, independent-sample $t$ tests were performed to look for differences in $\mathrm{rCBV}_{\max }$ and metabolite ratios between the 2 groups of oligodendrogliomas. Logistic regression analyses were performed to determine the significant independent predictor and to evaluate the association between $\mathrm{rCBV}_{\max }$ values and metabolite ratios to ascertain the best model to distinguish the 2 genotypes. To estimate the goodness of fit of the best logistic regression model, we performed the Hosmer-Lemeshow test. A $P$ value was computed from the $\chi^{2}$ distribution with $8 d f$ to test the fit of the logistic model. If the $P$ value was $>.05$, the model fit was considered acceptable. Receiver operating characteristic analyses were also used to ascertain the accuracy of the discriminatory models, and the area under the receiver operating characteristic curve at a 95\% confidence interval determined the accuracy. A Bonferroni correction was applied for analysis of the multivariate data involving 6 parameters $\left(\mathrm{rCBV}_{\text {max }}, \mathrm{NAA} / \mathrm{Cr}, \mathrm{Cho} / \mathrm{Cr}, \mathrm{Glx} / \mathrm{Cr}\right.$, myo-inositol/ $\mathrm{Cr}$, and lipid + lactate/Cr). To evaluate the robustness of multivariate logistic regression models, we also applied leave-one-out cross-validation tests.

Fisher exact $\chi^{2}$ tests were performed to evaluate the conventional MR imaging characteristics in distinguishing the 2 groups. All data analyses were performed with a statistical tool (SPSS for Windows, version 15.0; SPSS, Chicago, Illinois).

\section{RESULTS}

Representative images displaying the ${ }^{1} \mathrm{H}$-MR spectroscopy voxel corresponding to the $\mathrm{rCBV}_{\text {max }}$ region and spectra from patients with oligodendrogliomas harboring $1 \mathrm{p} / 19 \mathrm{q} \mathrm{LOH}$ and intact alleles are presented in Figs 1 and 2, respectively. $\mathrm{rCBV}_{\max }$ values and metabolite ratios between 2 genotypes of oligodendrogliomas are shown as box-and-whisker plots in Fig 3. Significant differences in $\mathrm{Cho} / \mathrm{Cr}(P=.046)$ between oligodendrogliomas with $1 \mathrm{p} / 19 \mathrm{q} \mathrm{LOH}$ and intact alleles were observed by use of the independent-sample $t$ test. No significant differences in $\mathrm{rCBV}_{\max }$ values and other ${ }^{1} \mathrm{H}$-MRS indices were observed between the 2 groups $(P>.05)$. 

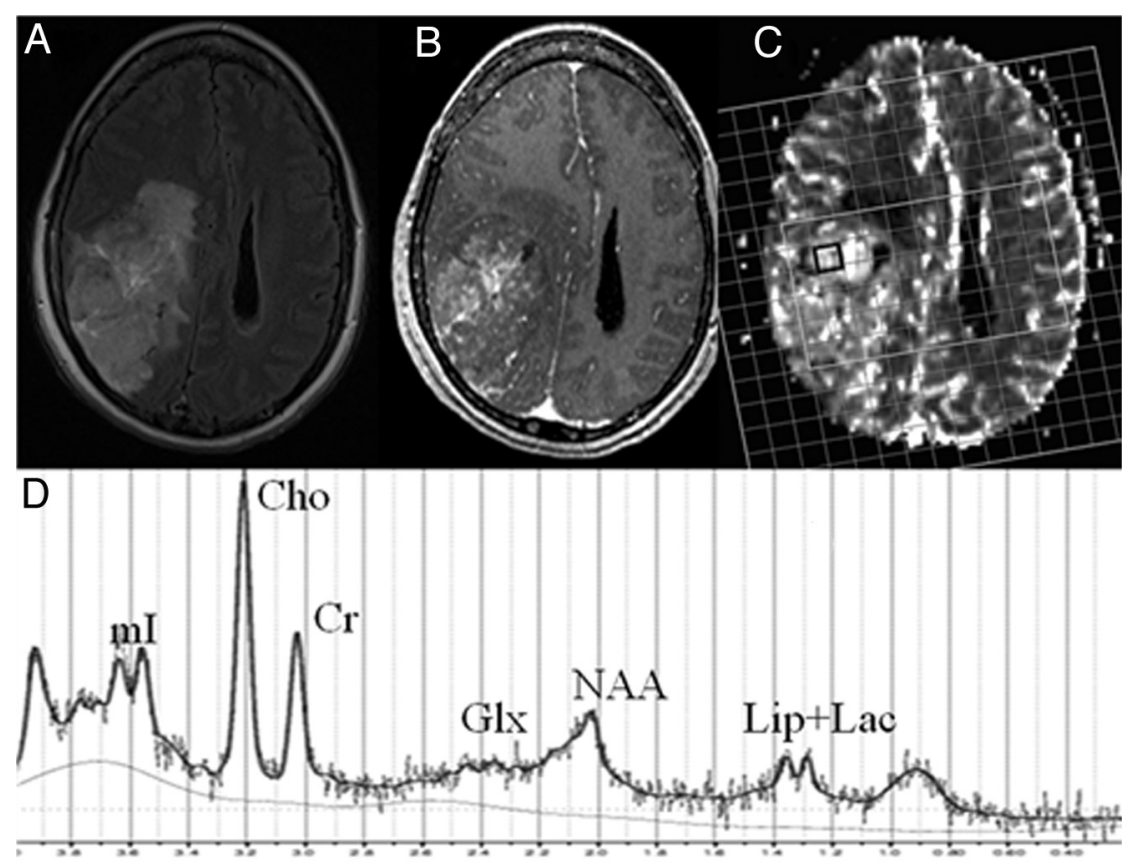

FIG 1. A 54-year-old woman with $1 p / 19 q \mathrm{LOH}$ oligodendroglioma. Axial FLAIR image (A) demonstrates an ill-defined hyperintense mass in the right parieto-occipital region extending into the lateral ventricles. There is heterogeneous contrast enhancement within the mass on the corresponding postcontrast T1-weighted image $(B)$. $^{1} \mathrm{H}-\mathrm{MRS}$ grid overlaid on CBV map $(C)$ demonstrating a voxel from the $\mathrm{rCBV}_{\max }$ region (black). Corresponding spectrum $(D)$ from this voxel shows lipid + lactate, NAA, Glx, Cr, Cho, and myo-inositol peaks.
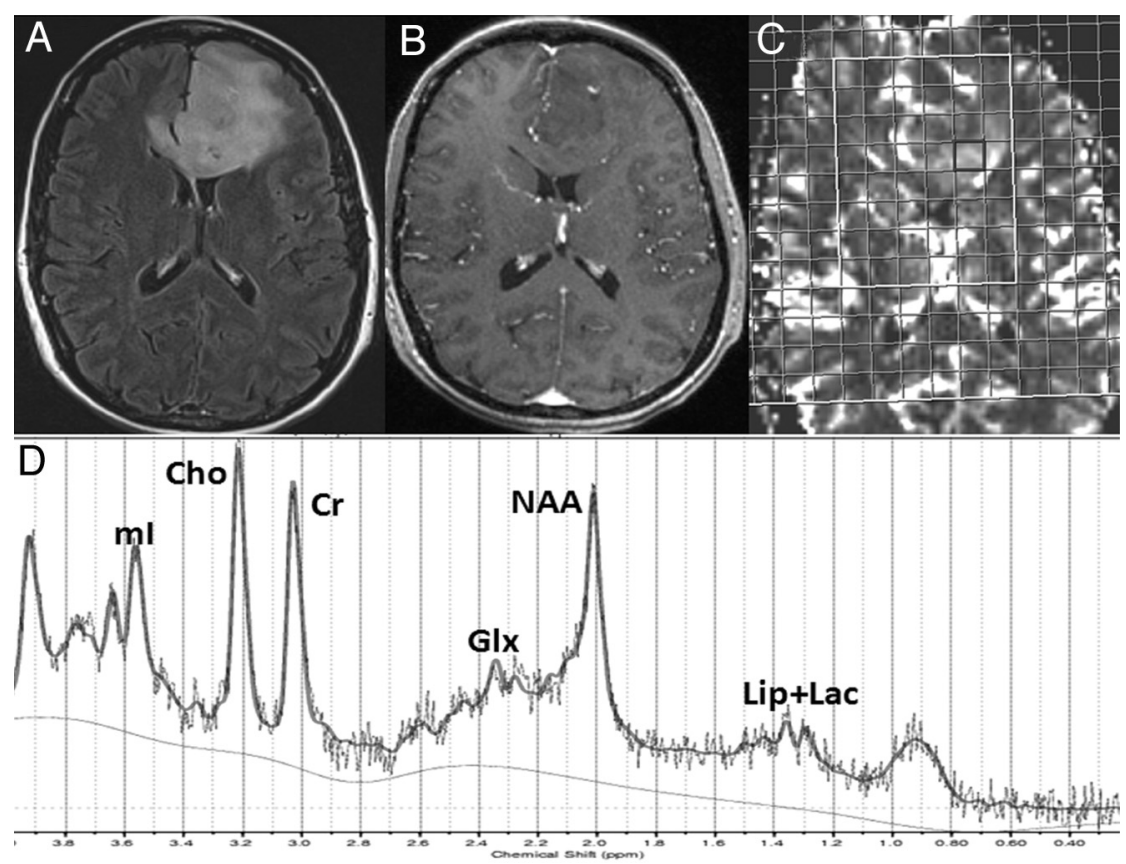

FIG 2. A 49-year-old woman diagnosed with oligodendroglioma with intact alleles. Axial FLAIR image $(A)$ demonstrates an extensive hyperintense mass in the left frontal lobe extending into the genu of the corpus callosum and lateral ventricles. There is a small nodular focus of enhancement within the mass on the corresponding postcontrast T1-weighted image (B). ${ }^{1} \mathrm{H}-\mathrm{MRS}$ grid overlaid on the CBV map (C) demonstrating a voxel from the $\mathrm{rCBV}_{\max }$ region (black). Corresponding spectrum $(D)$ displaying various metabolite resonances. Note the presence of a reduced $\mathrm{Cho} / \mathrm{Cr}$ ratio from $\mathrm{rCBV}_{\max }$ voxel compared with that seen in Fig 1.

When univariate logistic regression models were used to classify the genotypes, no single variable demonstrated significant contribution. However, among all of the variables tested in the univariate logistic regression model, the $\mathrm{Cho} / \mathrm{Cr}$ ratio had the best predictive value. Receiver operating characteristic analysis demonstrated moderate accuracy (area under the receiver operating characteristic curve, 0.68 ) of the Cho/Cr ratio (cutoff value, 0.41 ), with a sensitivity of $69.6 \%$ and a specificity of $64.2 \%$ (Fig 4). Among all bivariate logistic regression models, the $\mathrm{Cho} / \mathrm{Cr}$ ratio, together with the $\mathrm{rCBV}_{\text {max }}$ value, provided the best discriminatory classification with an accuracy (area under the receiver operating characteristic curve, 0.69 ), a sensitivity of $69.7 \%$, and a specificity of $70.6 \%$ (Fig 4). Accuracies for the bivariate models involving $\mathrm{rCBV}_{\max }$ and other metabolite ratios (NAA/Cr, myo-inositol/Cr, Glx/Cr, and lipid + lactate/Cr) were inferior to that of a model involving $\mathrm{rCBV}_{\max }$ and $\mathrm{Cho} / \mathrm{Cr}$ (data not shown). It is interesting to note that after incorporation of all of the metabolite ratios (NAA/Cr, Cho/Cr, Glx/Cr, myo-inositol/Cr, and lipid + lactate/Cr) and the $\mathrm{CBV}_{\text {max }}$ value in the multivariate logistic regression model, a significant improvement in the performance of the model was observed $(P=.018)$. A higher discriminative accuracy (area under the receiver operating characteristic curve, $0.72)$, sensitivity $(82.6 \%)$, and moderate specificity $(64.7 \%)$ were observed in distinguishing 2 groups of oligodendrogliomas (Fig 4). A $\chi^{2}$ value of 8.04 and a $P$ value of .429 were obtained from the Hosmer-Lemeshow test, reflecting an acceptable fit for the multivariate logistic regression model. Leave-one-out crossvalidation tests revealed that $70 \%$ of oligodendrogliomas were correctly classified into $1 \mathrm{p} / 19 \mathrm{q} \mathrm{LOH}$ and intact alleles by the multivariate logistic regression model.

\section{Conventional MR Imaging Characteristics}

Paramagnetic susceptibility effect was higher in patients with $1 \mathrm{p} / 19 \mathrm{q} \mathrm{LOH}$ oligodendrogliomas compared with those with intact alleles $(P=.04)$. Of the $23 \mathrm{pa}$ tients with $1 \mathrm{p} / 19 \mathrm{q} \mathrm{LOH}, 18$ patients demonstrated a susceptibility effect, whereas only 8 of 17 patients with intact alleles had a susceptibility effect. In addition, more patients with 1p/19q LOH oligodendrogliomas $(n=19 / 23)$ had an indistinct border on T2-weighted images than those with intact 

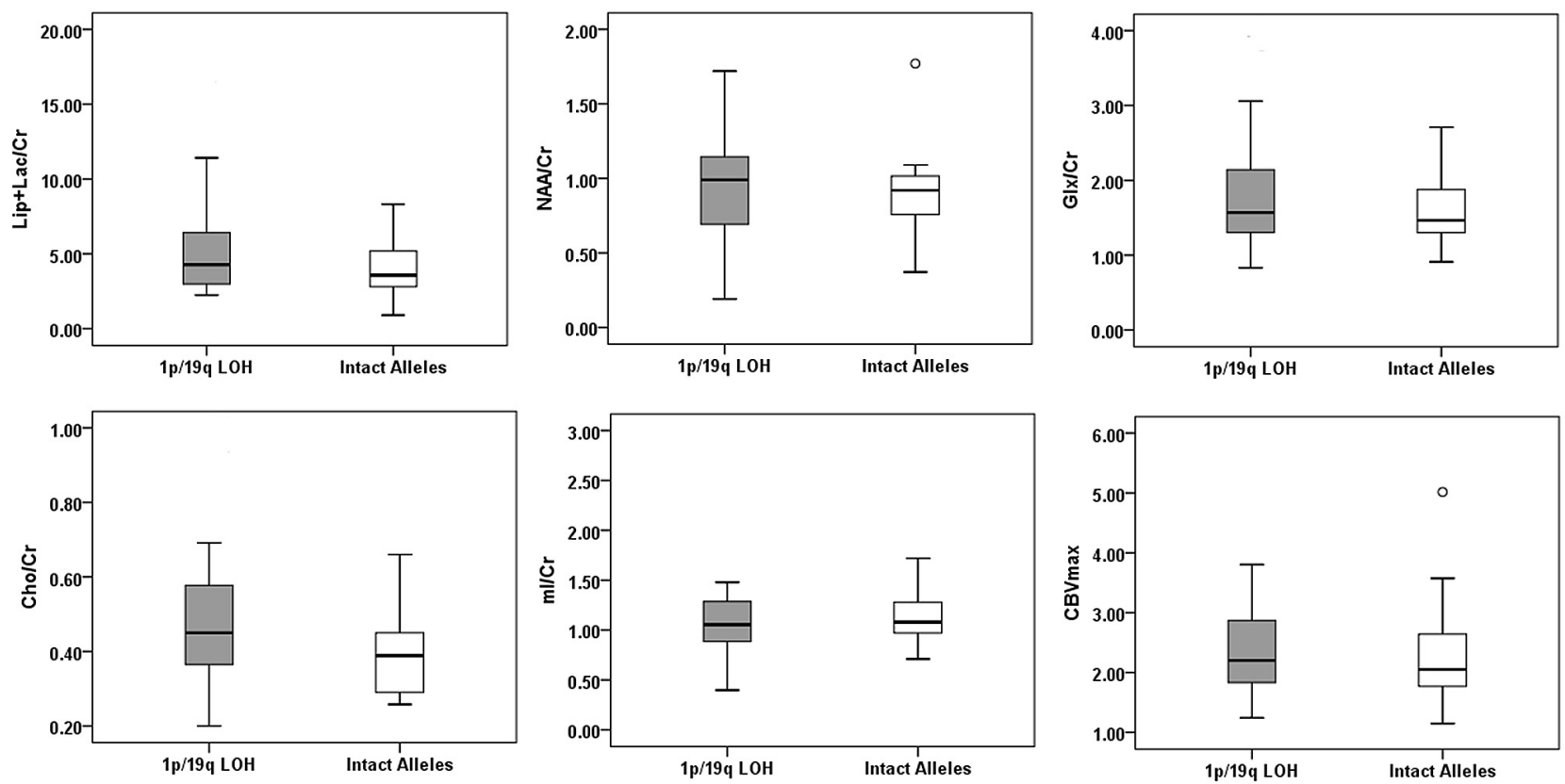

FIG 3. Box-and-whisker plots demonstrating the range of distribution of metabolite ratios from a voxel with $r C B V_{\max }$ in patients with $1 p / 19 q$ $\mathrm{LOH}$ (shaded) and intact alleles (white). Boxes represent the median, the $25^{\text {th }}$ percentile, and the $75^{\text {th }}$ percentile. Outlying values are marked with open circles. Cho/Cr was significantly different between the 2 genotypes of oligodendrogliomas.

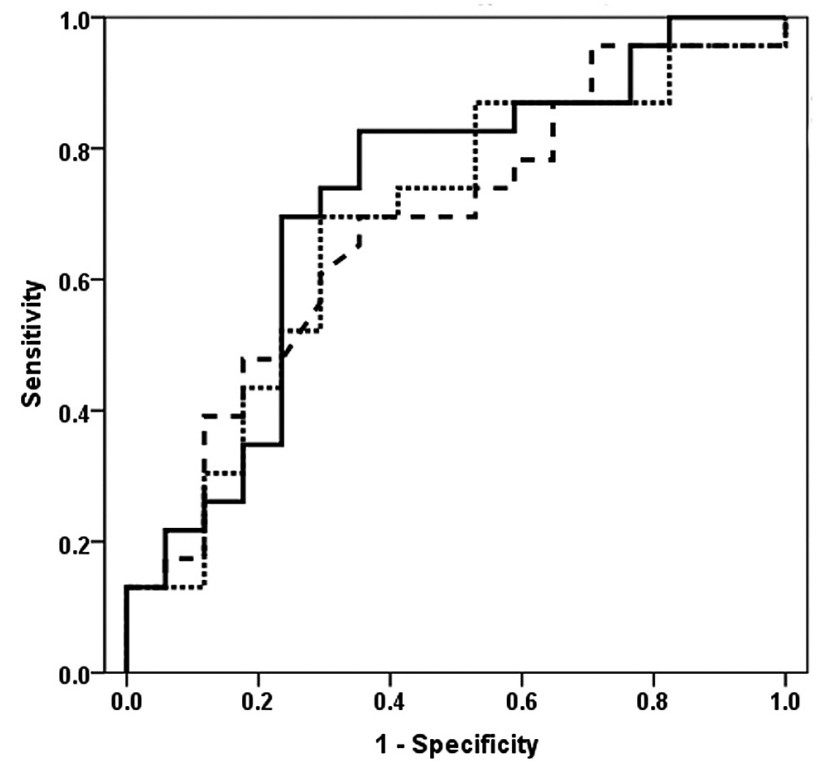

FIG 4. Receiver operating characteristic curves for $\mathrm{Cho} / \mathrm{Cr}$ ratio measured from $\mathrm{rCBV}_{\max }$ voxel exhibit an area under the receiver operating characteristic curve of 0.68 (broken line). The $\mathrm{Cho} / \mathrm{Cr}$ ratio, together with the $\mathrm{rCBV}_{\max }$ from the bivariate logistic regression analysis, shows an area under the receiver operating characteristic curve of 0.69 (dotted line). After incorporation of all of the metabolite ratios and $\mathrm{rCBV}_{\max }$ value in the multivariate logistic regression model, the area under the receiver operating characteristic curve is 0.72 (solid line). The multivariate logistic regression analysis provided the best model to distinguish the 2 groups of oligodendrogliomas, with a sensitivity of $82.6 \%$ and a specificity of $64.7 \%$. Leave-one-out cross-validation tests revealed that $70 \%$ of oligodendrogliomas were correctly classified.

alleles $(n=12 / 17)$, with a trend toward significance $(P=.07)$. However, contrast enhancement and signal intensity on T2- and
T1-weighted images did not differ between the 2 groups of oligodendrogliomas $(P>.05)$.

\section{DISCUSSION}

Our study demonstrates that the integration of $\mathrm{rCBV}_{\max }$ and ${ }^{1} \mathrm{H}$ MRS indices may aid in the discrimination of oligodendrogliomas with $1 \mathrm{p} / 19 \mathrm{q} \mathrm{LOH}$ from those with intact alleles. The prevalence of $1 \mathrm{p} / 19 \mathrm{q} \mathrm{LOH}$ in oligodendrogliomas is $50 \%-80 \%$, which is consistent with a $60 \%-70 \%$ response rate to procarbazine, vincristine, lomustine (PCV) ${ }^{24}$ whereas $50 \%-60 \%$ of patients respond to temozolomide. ${ }^{25}$ Although most anaplastic oligodendrogliomas are chemosensitive, the responsiveness of low-grade oligodendrogliomas and the most appropriate timing for therapy are unclear and debatable. ${ }^{26-28}$ On the contrary, some studies ${ }^{8,9}$ have demonstrated that oligodendrogliomas harboring $1 \mathrm{p} / 19 \mathrm{q} \mathrm{LOH}$, irrespective of histologic grade, present a favorable outcome to PCV chemotherapy, thus corroborating the need for a genetic profile to guide clinical management in such patients.

Previous studies have reported that both dynamic susceptibility contrast-PWI and arterial spin-labeling PWI can distinguish histologic grades of gliomas. ${ }^{29,30}$ However, PWI had limited usefulness in the differentiation of subgroups of oligodendrogliomas with considerable overlap in $\mathrm{rCBV}_{\max }$ and maximal cerebral blood flow between low and high grades. ${ }^{21,29,31}$ One study reported specific genotypic differences in oligodendrogliomas by use of dynamic susceptibility contrast-PWI, ${ }^{11}$ with significantly higher $\mathrm{rCBV}_{\max }$ in only low-grade oligodendrogliomas with $1 \mathrm{p} /$ $19 \mathrm{q} \mathrm{LOH} .{ }^{11}$ Although the precise mechanism for increased $\mathrm{rCBV}_{\text {max }}$ is unknown, it is believed that oligodendrogliomas with $1 \mathrm{p} / 19 \mathrm{q} \mathrm{LOH}$ are associated with increased metabolism, angiogenesis, and an intense vascular network. In a recent study, Kapoor et $\mathrm{al}^{12}$ reported significantly higher $\mathrm{rCBV}_{\max }$ in low-grade oligoden- 
drogliomas with $1 \mathrm{p} / 19 \mathrm{q} \mathrm{LOH}$ and increased vascular endothelial growth factor expression, CD31, and CD105, independent of epidermal growth factor receptor subtype, compared with tumors with intact alleles. In addition, significant associations between $\mathrm{rCBV}_{\max }$ with $1 \mathrm{p} / 19 \mathrm{q} \mathrm{LOH}$, and epidermal growth factor receptor and vascular endothelial growth factor expression were also reported in that study. Despite promising rCBV differences among genetically different low-grade oligodendrogliomas, previous studies ${ }^{11,12}$ did not observe a significant difference in $\mathrm{rCBV}_{\text {max }}$ between 2 genotypes among high-grade oligodendrogliomas. These studies exemplify the limitation of rCBV alone because a priori knowledge of WHO grading (via biopsy or surgical samples) would defeat the purpose of a noninvasive diagnostic imaging technique. Therefore, it is essential to explore the usefulness of other imaging biomarkers or a combination of biomarkers that can identify oligodendroglial genotypes before commencement of any treatment.

Numerous studies have shown the potential of ${ }^{1} \mathrm{H}-\mathrm{MR}$ spectroscopy in the evaluation of tumor grade ${ }^{15,32}$ and differentiation of tumor types. ${ }^{33,34}$ Recently, ${ }^{1} \mathrm{H}-\mathrm{MR}$ spectroscopy has also shown promise in determining the genotype profile of brain tumors. ${ }^{35}$ Nafe et $\mathrm{al}^{36}$ reported a significant correlation between $\mathrm{Cho} / \mathrm{Cr}$ and the degree of epidermal growth factor receptor in astrocytic tumors, suggesting that molecular analysis by ${ }^{1} \mathrm{H}-\mathrm{MR}$ spectroscopy may provide better characterization of brain tumors. Jenkinson et $\mathrm{al}^{10}$ observed a nonsignificant, but higher Cho/Cr ratio in oligodendrogliomas with $1 \mathrm{p} / 19 \mathrm{q} \mathrm{LOH}$ compared with patients with intact alleles. Given the heterogeneous nature of the neoplasm, it is possible that metabolite levels from a large single voxel (used in that study) might have included multiple tissue compartments because of partial volume averaging. This belief is also supported by a considerable overlap of ${ }^{1} \mathrm{H}-\mathrm{MR}$ spectroscopy indices between 2 grades of oligodendrogliomas. ${ }^{37}$ To address the tumor heterogeneity, Chawla et al ${ }^{16}$ used cerebral blood flow-guided analysis of ${ }^{1} \mathrm{H}$-MR spectroscopy and observed significantly higher $\mathrm{Cho} / \mathrm{Cr}$ and lipid + lactate/Cr from high-grade oligodendrogliomas compared with low-grade oligodendrogliomas.

Because the $\mathrm{rCBV}_{\max }$ region corresponds to greatest malignancy, ${ }^{21}$ we evaluated the Cho/Cr ratios from the voxel with the $\mathrm{rCBV}_{\text {max }}$ region and observed significantly different ratios between the 2 genotypes of oligodendrogliomas. However, no significant contribution of any single variable was observed in the univariate logistic regression models in distinguishing the 2 genotypes. This may be the result of tumor heterogeneity, which is prevalent in oligodendrogliomas. To the best of our knowledge, our study is the first that explores the usefulness of combining PWI-directed ${ }^{1} \mathrm{H}-\mathrm{MR}$ spectroscopy and PWI to distinguish 1p/ 19q LOH oligodendrogliomas from those with intact alleles. Because we focus on areas of high perfusion only, we believe that the differences (though small, but significant) are real and are not related to intratumoral heterogeneity. As ours is a single-institution study on a limited number of patients, future studies on larger cohorts may be necessary to evaluate the true clinical potential of our work.

When $\mathrm{rCBV}_{\text {max }}$ and Cho/Cr values were incorporated in a bivariate logistic regression analysis, a moderate sensitivity, spec- ificity, and accuracy was observed. However, incorporating $\mathrm{rCBV}_{\max }$ value and corresponding ${ }^{1} \mathrm{H}$-MRS indices as interaction components in a multivariate regression analysis provided the best accuracy. A relatively high sensitivity of $82.6 \%$ for this model indicates a high positive rate in discriminating the 2 groups of oligodendrogliomas. Given the inherently different physiologic and biochemical information that $\mathrm{rCBV}_{\text {max }}$ and ${ }^{1} \mathrm{H}$-MRS indices provide, we believe that these parameters may be complementary in tumor diagnosis. Multiparametric data analysis allows us to exploit the unique strengths of the different imaging techniques. As a combination of PWI and ${ }^{1} \mathrm{H}-\mathrm{MR}$ spectroscopy improves the diagnostic accuracy of gliomas, ${ }^{38}$ we used a similar approach to obtain greater discrimination of genotypes of oligodendrogliomas and showed that it was more sensitive than any single PWI/ ${ }^{1} \mathrm{H}-\mathrm{MR}$ spectroscopy parameter.

Cho-containing compounds (choline, phosphocholine, and glycerophosphocholine) are part of the normal phospholipid pathways that maintain cell membrane integrity. Higher Cho levels in tumors indicate increased membrane turnover because of cell proliferation or high cell density. ${ }^{39}$ Tumor vasculature plays a critical role in supplying nutrients and oxygen to cancer cells, providing a favorable environment for the cells to grow. This argument is supported by the fact that high rCBV correlates with increased Cho levels in gliomas. ${ }^{40}$ Histopathologic analysis shows that oligodendrogliomas with $1 \mathrm{p} / 19 \mathrm{q} \mathrm{LOH}$ grow as compact and hypercellular lesions, ${ }^{41}$ corroborating our observation of elevated levels of $\mathrm{Cho} / \mathrm{Cr}$ in these oligodendrogliomas. Higher proliferation of neoplastic cells and elevated metabolic rate lead to hypoxic regions; this cycle ultimately leads to energy failure and cell death that results in necrosis. We believe that elevated levels of lipid and lactate in the $1 \mathrm{p} / 19 \mathrm{q} \mathrm{LOH}$ oligodendrogliomas in our study is possibly attributed to a combined process of hypoxia, microscopic cellular necrosis, and cell proliferation in $\mathrm{rCBV}_{\max }$ regions of the neoplams. Both myo-inositol and Glx play important roles in the growth of oligodendrogliomas through multiple mechanisms. ${ }^{33,42}$ We believe that increased mitotic activity of neoplastic cells in the $\mathrm{rCBV}_{\text {max }}$ regions of $1 \mathrm{p} / 19 \mathrm{q} \mathrm{LOH}$ oligodendrogliomas may account for the increased myo-inositol and Glx levels.

Some studies ${ }^{22,23}$ have assessed the relationship between conventional MR imaging and genotypes of oligodendrogliomas. An indistinct tumor border, heterogeneous signal intensity, and paramagnetic susceptibility are associated with oligodendrogliomas harboring $1 \mathrm{p} / 19 \mathrm{q} \mathrm{LOH} .^{22,23}$ We observed a significantly higher susceptibility effect in oligodendrogliomas with $1 \mathrm{p} / 19 \mathrm{q}$ deletions. Calcification and tumor-associated hemorrhage are commonly seen in oligodendrogliomas, ${ }^{43}$ which may have contributed to the susceptibility effect in our study.

Previous studies ${ }^{22,23}$ have also reported that oligodendrogliomas harboring $1 \mathrm{p} / 19 \mathrm{q}$ loss usually demonstrate an indistinct border, whereas a sharp border is a characteristic of those with intact $1 \mathrm{p} / 19 \mathrm{q}$ alleles. We also observed an indistinct border more frequently in oligodendrogliomas with $1 \mathrm{p} / 19 \mathrm{q} \mathrm{LOH}$ compared with intact alleles; however, the difference was not significant. Furthermore, unlike previous reports, ${ }^{22}$ no significant difference in signal intensity of T2- and T1-weighted images was observed in our study between 2 groups of oligodendrogliomas. As morphologic imaging characteristics are usually evaluated qualitatively and are 
observer-dependent, assessment of imaging features is subjective and may have considerable user bias. We believe that the discrepancy between our results and those published in the literature may be attributed to observer variability in assessing these imaging characteristics. These observations further imply that evaluation of conventional imaging features alone may not be a reliable approach in distinguishing genotypes of oligodendrogliomas. On the contrary, techniques such as PWI and ${ }^{1} \mathrm{H}-\mathrm{MR}$ spectroscopy are physiologically sensitive and quantitative and consequently may be more reliable.

Our study was limited by the relatively small sample size, as we had to exclude 10 patients because of unavailability of a cytogenetic profile and suboptimal PWI or ${ }^{1} \mathrm{H}-\mathrm{MRS}$ data. The second limitation of our study was a certain degree of heterogeneity in our patient population, as we also included patients with oligoastrocytomas. In general, oligoastrocytomas exhibit p53 deletions and $17 \mathrm{p} \mathrm{LOH}$ that are associated with astrocytic biologic behavior. However, allelic loss on chromosomes $1 \mathrm{p}$ and $19 \mathrm{q}$ is also exhibited by oligoastrocytomas, suggesting a monoclonal origin of oligodendrogliomas and oligoastrocytomas. Similar to pure oligodendrogliomas, patients with oligoastrocytomas also exhibit favorable prognosis and clinical response to chemoradiation therapy. ${ }^{7}$ On the basis of similarities in genetic aberrations and clinical features, oligodendrogliomas and oligoastrocytomas are often grouped together as oligodendroglial tumors; thus, their inclusion in the current study is justifiable.

Another limitation of our study was limited tissue coverage, as we used a single-section ${ }^{1} \mathrm{H}-\mathrm{MRS}$ to coregister rCBV maps; thus, only a part of the tumor was analyzed. To cover the whole neoplasm, or at least the bulk of the tumor volume, it is desirable to use a $3 \mathrm{D}{ }^{1} \mathrm{H}$-MRS sequence. However, such techniques lead to increased acquisition time that could be prohibitive in routine clinical settings. Parallel MRS with sensitivity encoding or generalized autocalibrated partially parallel acquisitions or echo-planar spectroscopic imaging techniques can overcome this problem.

Leakage of the gadolinium-based contrast agents in neoplastic lesions from the vascular compartment to the interstitial space may lead to underestimation of rCBV values. ${ }^{44,45}$ To account for this error, a preloading dose of contrast agent was administered 3 minutes before the contrast bolus to acquire PWI data as has been reported previously. ${ }^{18,46}$ Although largely successful, the resulting signal might still be contaminated by a T1effect that can be accounted for by mathematical modeling. Although we did not perform such corrections, we believe that this potential error would cancel out because the assumed underestimation of CBV would have affected both of the patient groups.

\section{CONCLUSIONS}

Our study demonstrates that integration of ${ }^{1} \mathrm{H}-\mathrm{MRS}$ indices from the $\mathrm{rCBV}_{\max }$ voxel may be helpful in distinguishing oligodendrogliomas with $1 \mathrm{p} / 19 \mathrm{q} \mathrm{LOH}$ from those with intact alleles. However, further studies in a larger patient population are required to confirm our findings.

Disclosures: Jaroslaw Krejza—UNRELATED: Board Membership: Neuroradiology Journal, Comments: Editor-in-Chief. Donald O'Rourke-UNRELATED: Consultancy: Merrill-Lynch, Comments: company review (biotechnology), unrelated to this man- uscript; Expert Testimony: Medico-legal consultancy, Comments: clinical activity expert review only, no research; Grants/Grants Pending: National Institutes of Health, ${ }^{*}$ Celldex Therapeutics; ${ }^{*}$ Patents (planned, pending, or issued): Trustee: University of Pennsylvania, ${ }^{*}$ Comments: US and Europe approved patent: Methods and Compositions for Treating Tumors (ErbB inhibition and Radiotherapy for Cancer); Royalties: see above patent. ${ }^{*}$ Harish Poptani-UNRELATED: Consultancy: American College of Radiology Image Metrix, Comments: consultant for a liver spectroscopy project, which is not relevant to this study; Grants/Grants Pending: National Institutes of Health. * ( ${ }^{\star}$ Money paid to institution.)

\section{REFERENCES}

1. Engelhard HH, Stelea A, Cochran EJ. Oligodendroglioma: pathology and molecular biology. Surg Neurol 2002;58:111-17

2. Kleihues P, Louis DN, Scheithauer BW, et al. The WHO classification of tumors of the nervous system. J Neuropathol Exp Neurol 2002;61: 215-25

3. Bromberg JE, van den Bent MJ. Oligodendrogliomas: molecular biology and treatment. Oncologist 2009;14:155-63

4. Bigner SH, Rasheed BK, Wiltshire R, et al. Morphologic and molecular genetic aspects of oligodendroglial neoplasms. Neuro Oncol 1999;1:52-60

5. Aldape K, Burger PC, Perry A. Clinicopathologic aspects of 1p/19q loss and the diagnosis of oligodendroglioma. Arch Pathol Lab Med 2007;131:242-51

6. Hartmann C, von Deimling A. Molecular pathology of oligodendroglial tumors. Recent results. Cancer Res 2009;171:25-49

7. Cairncross JG, Ueki K, Zlatescu MC, et al. Specific genetic predictors of chemotherapeutic response and survival in patients with anaplastic oligodendrogliomas. J Natl Cancer Inst 1998;90:1473-79

8. Iwamoto FM, Nicolardi L, Demopoulos A, et al. Clinical relevance of $1 p$ and $19 q$ deletion for patients with WHO grade 2 and 3 gliomas. J Neurooncol 2008;88:293-98

9. Walker C, Haylock B, Husband D, et al. Clinical use of genotype to predict chemosensitivity in oligodendroglial tumors. Neurology 2006;66:1661-67

10. Jenkinson MD, Smith TS, Joyce K, et al. MRS of oligodendroglial tumors: correlation with histopathology and genetic subtypes. Neurology 2005;64:2085-89

11. Whitmore RG, Krejza J, Kapoor GS, et al. Prediction of oligodendroglial tumor subtype and grade using perfusion weighted magnetic resonance imaging. J Neurosurg 2007;107:600-09

12. Kapoor GS, Gocke TA, Chawla S, et al. Magnetic resonance perfusion-weighted imaging defines angiogenic subtypes of oligodendroglioma according to 1p19q and EGFR status. J Neurooncol 2009; 92:373-86

13. Spampinato MV, Smith JK, Kwock L, et al. Cerebral blood volume measurements and proton MR spectroscopy in grading of oligodendroglial tumors. AJR Am J Roentgenol 2007;188:204-12

14. Daumas-Duport C, Tucker ML, Kolles H, et al. Oligodendrogliomas. Part II: A new grading system based on morphological and imaging criteria. J Neurooncol 1997;34:61-78

15. Law M, Yang S, Wang H, et al. Glioma grading: sensitivity, specificity, and predictive values of perfusion MR imaging and proton MR spectroscopic imaging compared with conventional MR imaging. AJNR Am J Neuroradiol 2003;24:1989-98

16. Chawla S, Wang S, Wolf RL, et al. Arterial spin-labeling and MR spectroscopy in the differentiation of gliomas. AJNR Am J Neuroradiol 2007;28:1683-89

17. Hatanpaa KJ, Burger PC, Eshleman JR, et al. Molecular diagnosis of oligodendroglioma in paraffin sections. Lab Invest 2003;83:419-28

18. Morita N, Wang S, Chawla S, et al. Dynamic susceptibility contrast perfusion weighted imaging in grading of nonenhancing astrocytomas. J Magn Reson Imaging 2010;32:803-08

19. Cha S, Knopp EA, Johnson G, et al. Intracranial mass lesions: dynamic contrast-enhanced susceptibility-weighted echo-planar perfusion MR imaging. Radiology 2002;223:11-29

20. Provencher SW. Automatic quantitation of localized in vivo $\mathbf{1 H}$ spectra with LCModel. NMR Biomed 2001;14:260-64

21. Lev MH, Ozsunar Y, Henson JW, et al. Glial tumor grading and 
outcome prediction using dynamic spin-echo MR susceptibility mapping compared with conventional contrast-enhanced MR: confounding effect of elevated rCBV of oligodendrogliomas. AJNR Am J Neuroradiol 2004;25:214-21

22. Megyesi JF, Kachur E, Lee DH, et al. Imaging correlates of molecular signatures in oligodendrogliomas. Clin Cancer Res 2004;10:4303-06

23. Jenkinson MD, du Plessis DG, Smith TS, et al. Histological growth patterns and genotype in oligodendroglial tumours: correlation with MRI features. Brain 2006;129:1884-91

24. Cairncross JG, Macdonald DR. Successful chemotherapy for recurrent malignant oligodendroglioma. Ann Neurol 1988;23:360-64

25. van den Bent MJ, Taphoorn MJ, Brandes AA, et al. Phase II study of first-line chemotherapy with temozolomide in recurrent oligodendroglial tumors: the European Organization for Research and Treatment of Cancer Brain Tumor Group Study 26971. J Clin Oncol 2003;21:2525-28

26. Engelhard HH, Stelea A, Mundt A. Oligodendroglioma and anaplastic oligodendroglioma: clinical features, treatment, and prognosis. Surg Neurol 2003;60:443-56

27. van den Bent MJ. Can chemotherapy replace radiotherapy in lowgrade gliomas? Time for randomized studies. Semin Oncol 2003; 30(6 Suppl 19):39-44

28. Iwadate $Y$, Matsutani $T$, Hasegawa $Y$, et al. Favorable long-term outcome of low-grade oligodendrogliomas irrespective of $1 \mathrm{p} / 19 \mathrm{q}$ status when treated without radiotherapy. J Neurooncol 2011;102: $443-49$

29. Wolf RL, Wang J, Wang S, et al. Grading of CNS neoplasms using continuous arterial spin labeled perfusion MR imaging at 3 Tesla. $J$ Magn Reson Imaging 2005;22:475-82

30. Di Costanzo A, Pollice S, Trojsi F, et al. Role of perfusion-weighted imaging at 3 Tesla in the assessment of malignancy of cerebral gliomas. Radiol Med 2008;113:134-43

31. Xu M, See SJ, Ng WH, et al. Comparison of magnetic resonance spectroscopy and perfusion-weighted imaging in presurgical grading of oligodendroglial tumors. Neurosurgery 2005;56:919-26

32. Hsu YY, Chang CN, Wie KJ, et al. Proton magnetic resonance spectroscopic imaging of cerebral gliomas: correlation of metabolite ratios with histopathologic grading. Chang Gung Med J 2004;27:399407

33. Chawla S, Oleaga L, Wang S, et al. Role of proton magnetic resonance spectroscopy in differentiating oligodendrogliomas from astrocytomas. J Neuroimaging 2010;20:3-8
34. Chawla S, Zhang Y, Wang S, et al. Proton magnetic resonance spectroscopy in differentiating glioblastomas from primary cerebral lymphomas and brain metastases. J Comput Assist Tomogr 2010;34: 836-41

35. Thorsen F, Jirak D, Wang J, et al. Two distinct tumor phenotypes isolated from glioblastomas show different MRS characteristics. NMR Biomed 2008;21:830-38

36. Nafe R, Glienke W, Hattingen E, et al. Correlation between amplification of the gene for the epidermal growth factor receptor (EGFR), data from preoperative proton-MR-spectroscopy (1HMRS) and histomorphometric data of glioblastomas. Anal Quant Cytol Histol 2007;29:199-207

37. Tate AR, Majos C, Moreno A, et al. Automated classification of short echo time in in vivo $1 \mathrm{H}$ brain tumor spectra: a multicenter study. Magn Reson Med 2003;49:29-36

38. Di Costanzo A, Scarabino T, Trojsi F, et al. Multiparametric 3T MR approach to the assessment of cerebral gliomas: tumor extent and malignancy. Neuroradiology 2006;48:622-31

39. Miller BL. A review of chemical issues in $1 \mathrm{H}$ NMR spectroscopy: N-acetyl-L-aspartate, creatine and choline. NMR Biomed 1991;4: 47-52

40. Henry RG, Vigneron DB, Fischbein NJ, et al. Comparison of relative cerebral blood volume and proton spectroscopy in patients with treated gliomas. AJNR Am J Neuroradiol 2000;21:357-66

41. Palfi S, Swanson KR, De Bouard S, et al. Correlation of in vitro infiltration with glioma histological type in organotypic brain slices. Br J Cancer 2004;91:745-52

42. de Groot J, Sontheimer H. Glutamate and the biology of gliomas. Glia 2011;59:1181-89

43. Lee YY, Van Tassel P. Intracranial oligodendrogliomas: imaging findings in 35 untreated cases. AJR Am J Roentgenol 1989;152: 361-69

44. Schmainda KM, Rand SD, Joseph AM, et al. Characterization of a first-pass gradient-echo spin-echo method to predict brain tumor grade and angiogenesis. AJNR Am J Neuroradiol 2004;25:1524-32

45. Quarles CC, Ward BD, Schmainda KM. Improving the reliability of obtaining tumor hemodynamic parameters in the presence of contrast agent extravasation. Magn Reson Med 2005;53:1307-16

46. Donahue KM, Krouwer HG, Rand SD, et al. Utility of simultaneously acquired gradient-echo and spin-echo cerebral blood volume and morphology maps in brain tumor patients. Magn Reson Med 2000;43:845-53 